Z Rheumatol 2021 · 80:1

https://doi.org/10.1007/s00393-020-00958-1

Angenommen: 19. Dezember 2020

(c) Springer Medizin Verlag $\mathrm{GmbH}$, ein Teil von Springer Nature 2021

Die Rheumatologie hat in den letzten 20 Jahren mit der Einführung neuer diagnostischer Verfahren und den modernen zielgerichteten Therapiemöglichkeiten einen grundlegenden Wandel durchlaufen. Während hierdurch die Behandlungsoptionen von Patienten mit rheumatischen Erkrankungen wesentlich erweitert und die Prognose verbessert werden konnten, sind im gleichen Zeitraum die Erfolge bei der Ausbildung und Weiterbildung im Gebiet der Rheumatologie begrenzt geblieben. Trotz großer Anstrengungen konnte die Zahl der rheumatologischen Lehrstühle und unabhängigen Professuren nicht wesentlich gesteigert werden, weshalb die Rheumatologie an den deutschen Universitäten weiterhin unterrepräsentiert bleibt. Dies wirkt sich vielerorts direkt auf die studentischen Lehrpläne aus, in denen rheumatologische Themen nur unzureichend berücksichtigt werden. Zudem hat die zunehmende Ökonomisierung in der Medizin zum Verlust von Weiterbildungsstellen in der Rheumatologie geführt, was die Nachwuchsproblematik zusätzlich verschärft.

\section{/) Die Rheumatologie an den deutschen Universitäten bleibt weiterhin unterrepräsentiert}

Wie im Memorandum der DGRh ausgeführt, besteht in weiten Teilen Deutschlands eine Unterversorgung mit Rheumatologinnen und Rheumatologen [1]. Für die Verbesserung der Versorgungssituation ist deshalb eine steigende Anzahl von gut ausgebildeten und motivierten jungen Rheumatologinnen und Rheumatologen von entscheidender Bedeutung,

\author{
M. Fleck ${ }^{1,2} \cdot$ B. F. Hoyer ${ }^{3}$ \\ ${ }^{1}$ Klinik und Poliklinik für Innere Medizin I, Universitätsklinikum Regensburg, Regensburg, Deutschland \\ ${ }^{2}$ Klinik und Poliklinik für Rheumatologie/Klinische Immunologie, Asklepios Klinikum Bad Abbach, Bad \\ Abbach, Deutschland \\ ${ }^{3}$ Klinik für Innere Medizin I, Rheumatologie/klinische Immunologie und Exzellenzzentrum \\ Entzündungsmedizin, Universitätsklinikum Schleswig-Holstein, Campus Kiel, Kiel, Deutschland
}

\title{
Der Weg in die Rheumatologie
}

die auch für die zukünftige Sicherung unseres Fachgebietes im Kanon der internistischen Schwerpunkte unerlässlich sind. Vor diesem Hintergrund ist der Themenschwerpunkt „Wege in die Rheumatologie“ als eine Bestandsaufnahme zu verstehen, die zur Diskussion anregen und als Grundlage für notwendige Verbesserungen in der rheumatologischen Aus- und Weiterbildung dienen soll.

In 4 Übersichtarbeiten wird der $\mathrm{Zu}$ gang zur Rheumatologie ausgehend vom Studium und über die Weiterbildung aufgezeigt. Es werden in diesen Artikeln wichtige Aspekte zur studentischen Lehre und der fachärztlichen Weiterbildung nach der neuen Musterweiterbildungsordnung dargestellt und die dabei bestehenden Herausforderungen und Probleme klar benannt. Auch der besondere Stellenwert der akademischen Rheumatologie und wie eine akademische Karriere gelingen kann sowie die rheumatologische Weiterbildungssituation aus der Sicht der Weiterbilder werden thematisiert.

Wir danken allen Autorinnen und Autoren für diese gelungenen Beiträge, die diese Bestandsaufnahme ermöglichen und den Weg für Verbesserungen in der rheumatologischen Ausund Weiterbildung weisen. Wir wünschen Ihnen eine angeregte Lektüre und würden uns sehr freuen, wenn die Übersichtsartikel Diskussionen auch in Ihrem Umfeld anstoßen würden.
Beste Grüße,

Ihre

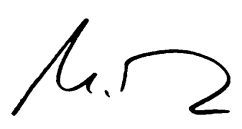

M. Fleck

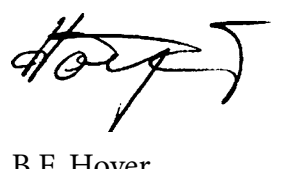

\section{Korrespondenzadresse}

Prof. Dr. med. M. Fleck

Klinik und Poliklinik für Innere Medizin I, Universitätsklinikum Regensburg

Franz-Josef-Strauß-Allee 11, 93053 Regens-

burg, Deutschland

martin.fleck@ukr.de

\section{Prof. Dr. B. F. Hoyer}

Klinik für Innere Medizin I, Rheumatologie/ klinische Immunologie und Exzellenzzentrum Entzündungsmedizin, Universitätsklinikum Schleswig-Holstein, Campus Kiel Arnold-Heller-Str. 3, 24105 Kiel, Deutschland Bimba.Hoyer@uksh.de

Interessenkonflikt. M. Fleck und B.F. Hoyer geben an, dass kein Interessenkonflikt besteht.

\section{Literatur}

1. Zink A, Braun J, Gromnica-Ihle E, Krause D, Lakomek HJ, Mau W, Müller-Ladner U, Rautenstrauch J, Specker C, Schneider M (2017) Memorandum der Deutschen Gesellschaft für Rheumatologie zur Versorgungsqualität in der Rheumatologie Update 2016.ZRheumatol 76:195-207 